LA-UR- $97-3762$

EA-UR=-9X-XXXX

Approved for public release;

distribution is unlimited

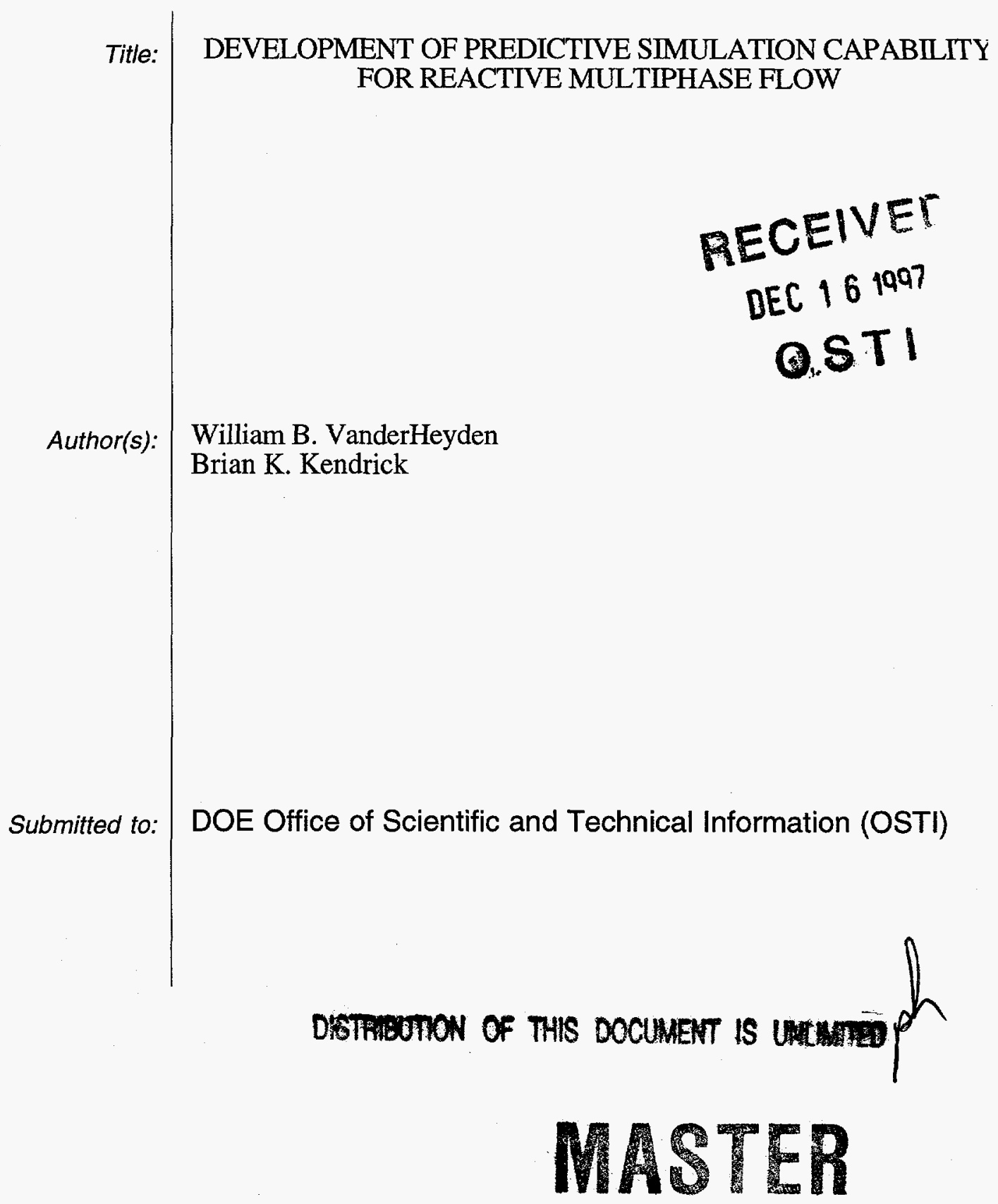




\section{DISCLAIMIER}

Portions of this docoment may be Illegible in electronic image products. Insges are produced from the best available original document 


\section{DISCLAIMER}

This report was prepared as an account of work sponsored by an agency of the United States Government. Neither the United States Government nor any agency thereof, nor any of their employees, makes any warranty, express or implied, or assumes any legal liability or responsibility for the accuracy, completeness, or usefulness of any information, apparatus, product, or process disclosed, or represents that its use would not infringe privately owned rights. Reference herein to any specific commercial product, process, or service by trade name, trademark, manufacturer, or otherwise does not necessarily constitute or imply its endorsement, recommendation, or favoring by the United States Government or any agency thereof. The views and opinions of authors expressed herein do not necessarily state or reflect those of the United States Government or any agency thereof. 


\title{
DEVELOPMENT OF PREDICTIVE SIMULATION CAPABILITY FOR REACTIVE MULTIPHASE FLOW
}

\author{
William B. VanderHeyden * \\ Brian K. Kendrick \\ Theoretical Division \\ Los Alamos National Laboratory
}

\begin{abstract}
This is the final report of a proposed three-year, Laboratory-Directed Research and Development (LDRD) project at the Los Alamos National Laboratory (LANL). The project was terminated after the first year due to changes in funding priorities. The objective of the project was to develop a self-sustained research program for advanced computer simulation of industrial reactive multiphase flows. The prototype research problem was a three-phase alumina precipitator used in the Bayer process, a key step in aluminum refining. Accomplishments in the first year included the development of an improved reaction mechanism of the alumina precipitation growth process, the development of an efficient method for handling particle size distribution in multiphase flow simulation codes and finally the incorporation of precipitation growth and agglomeration kinetics in LANL's CFDLIB multiphase flow code library.
\end{abstract}

\section{Background and Research Objectives}

Reactive multiphase flows are found in many of the processes across the spectrum of manufacturing industries. Examples of interpenetrating multiphase flows include riser reactors used in petroleum refining, bubble column reactors used in chemical manufacturing and sparged aqueous precipitators used in aluminum production.

In most cases, control of the spatial distribution of the individual phases of the multiphase flow can have a large impact on the efficiency of the processes. With the recent advances in the size and speed of modern supercomputers and with the continuing improvement in the state-of-theart of the mathematical description of reactive multiphase flows, it is becoming possible to use computer simulation to scale-up, optimize and design industrial multiphase flow equipment more accurately and precisely than is possible with traditional methods. Due to the complexity of multiphase flows and also to the intensive nature of the computer calculations used to solve

\footnotetext{
${ }^{*}$ Principal Investigator: e-mail: wbv@lanl.gov
} 
their governing equations, however, on-going research is needed to improve both the mathematical description and the numerical solution of the multiphase flow equations.

In order to extend the state-of-the-art and to develop a sustained laboratory research and development program, we sought to develop reactive multiphase flow simulations of a prototype problem from the aluminum industry. The prototype problem was a simulation of the three-phase alumina precipitator used in the Bayer process. The simulation was to involve the coupling of reactive chemistry modeling with fluid dynamics simulation. The following gives a brief description of this process and how reactive multiphase flow simulation might identify improvements.

Bayer Process Alumina Precipitators: Aluminum production from bauxite ore involves the electrolytic reduction of purified alumina $\left(\mathrm{Al}_{2} \mathrm{O}_{3}\right)$ to metallic aluminum. Virtually all aluminum refining operations use the Bayer process to convert raw bauxite ore to purified alumina [1]. A key step in the Bayer process is the precipitation of supersaturated caustic solutions of alumina to produce agglomerated hydrated alumina particles. The precipitation reaction involves 4 mechanisms: nucleation, growth, agglomeration and breakage [2]. Nucleation involves the formation of small alumina seed particles upon which further alumina is deposited via a surface reaction [2]. Supersaturated alumina solutions have a high apparent stability so it is necessary to assist the nucleation process by seeding the alumina solution with hydrated alumina fines. Particles produced from nucleation and growth can also agglomerate. Such agglomerates can also break up under the influence of agitation. High supersaturation and low agitation rates favor agglomeration [2].

The precipitation process is operated with two prime objectives: a cost-effective high recovery of product and a proper size distribution of particles [1,2]. The capacity of the alumina plant depends on recovery efficiency. Excess fines can cause problems in downstream filtration, clarification and calcination equipment [1,2]. These two objectives are, however, generally in conflict. High recoveries can be obtained with the production of excess fines. Desirable size distributions can be obtained but with poor recovery [2]. Precipitator design must balance these two objectives by effectively managing the multiphase flow, the precipitation kinetics and their interactions.

Because the surface growth reaction is very slow, high residence times (35-75 hours) are required in industrial equipment [2]. In an industrial alumina plant, precipitation reactions are performed in cylindrical tanks with diameters up to ten meters and heights typically two-and-a- 
half to three times the diameter [1]. Usually, anywhere from six to thirteen of these tanks are connected in series by troughs at the top [1]. As a result, the precipitation equipment represents a significant portion of the capital investment required for the Bayer process and its optimization carries significant incentives.

The precipitator vessels are also reasonably complicated. In older designs, air-lift or gas sparging is used in conjunction with an internal draught tube to circulate the seed and slurry in the vessel. These air-lift designs include a conical bottom to help collect the seed for recirculation [1]. Newer designs use turbine agitators inside a large draught tube to promote circulation [1]. In these designs, flow proceeds down the draught tube and up through the annular portion of the tanks. The upflow velocity can be used to control the maximum size of the seed particles through their settling velocity. Baffles can also be used to optimize the flow patterns in the precipitator [3]. The most desirable mode of operation would be to advance large particles through the tanks while retaining the finer particles for further precipitation [1,3].

The precipitation and agglomeration processes are coupled to the complex fluid dynamics of the solution in the precipitation tanks because the velocity distributions dictate residence times. The velocity fields, in turn, depend on the particle distributions and reaction extents. Finding optimal precipitator vessel designs which properly balance the two prime objectives through management of the coupling of multiphase flow and precipitation kinetics should be aided substantially by a detailed three-dimensional simulation of these processes. Such a capability could be used in a similar vein on problems in a number of industries including petroleum refining and chemicals manufacturing. Development of this capability is the long-term objective of this project.

\section{Importance to LANL's Science and Technology Base and National R\&D Needs}

Advances made in the area of reactive multiphase flow simulation should help to strengthen at least two Los Alamos National Laboratory core competencies. Most obviously, LANL's core competency in the area of Theory, Modeling and High-Performance Computing should benefit directly from improvements made in multiphase flow physics and precipitation chemistry. In addition, the advances made from the optimization of the LANL's CFDLIB multiphase flow codes for advanced workstation and parallel computing environments should also contribute to this competency. 
LANL's competency in the area of Nuclear and Advanced Materials should also benefit from advancements from this project. Multiphase precipitators are used in the recovery of plutonium from waste streams at LANL's TA-55 Plutonium Facility.

The goals of this project also reinforce some of DOE's key strategies including Industrial Competitiveness and Science and Technology.

\section{Scientific Approach and Accomplishments}

This project was divided into two major work components. The first component focused on the development of a coupled reactive multiphase flow simulation of the Bayer process alumina precipitator. The second component focused on validating and improving precipitation and agglomeration reaction kinetics models from the open literature and on the issues of coupling the improved kinetics calculations to the multiphase flow simulation.

A significant challenge in the development of a reactive multiphase flow simulation of the alumina precipitator is posed by the disparity of time scales and the computationally intensive nature of the fluid flow simulations. The time scale of the precipitation and agglomeration reactions in this problem are long--many hours. On the other hand, the time scale of the fluid dynamics in the alumina precipitator can be quite short. For example, the time scale associated with the gas jet is on the order of seconds. Furthermore, a highly resolved three-dimensional simulation of the fluid dynamics alone can take many CPU-hours on today's advanced work stations and supercomputers. Performing such simulations over the long precipitation and agglomeration reaction time scale would be prohibitive. As a result, approximate methods and compromises had to be developed to obtain a practical yet reasonably accurate coupled reactive multiphase flow simulation. The scientific approach is outlined below.

Scientific Approach: Two approximate methods were proposed to contend with the challenges posed by the wide disparity of time scales in the alumina precipitator simulation problem. We term these two approaches weak and coarse resolution direct coupling.

A weakly coupled simulation is one in which time-dependent fluid flow calculations are first performed assuming no chemical reactions occur with an assumed steady-state distribution of precipitator reactant and products. Once the time-dependent fluid motion reaches a statistical steady state, a simulated tracer is introduced into the feed of the precipitator. A residence time distribution function (a residence time distribution is the statistical distribution of fluid packet 
ages in the precipitator) is constructed by recording the tracer concentration in the precipitator outflow as a function of time. From the residence time distribution, a reaction kinetics calculation is performed to obtain average reaction extents. Base on the results of the reaction kinetics calculation, the initial guess for the distribution of reactants and products used in the flow simulation is revised and the fluid flow simulation is repeated. This iterative procedure is continued until convergence is obtained. The coupling between the reaction kinetics and the fluid flow is weak in this method since only average information (the residence time distribution and the average reactant extent) is communicated between the fluid flow simulation and the reaction kinetics calculations. All local information is lost. The hope, however, is that this method is much faster than a fully coupled simulation and provides reasonable accuracy.

A directly coupled calculation is one in which the equations of motion for the fluids and the reaction kinetics equations are solved simultaneously in a cell-by-cell fashion every time step. As discussed previously, a fully resolved directly coupled calculation is computationally prohibitive. Some approximations can be introduced, however, to lower cost. The approximations might include the use of coarse time and space resolution or the use of a fully implicit scheme for the integration of the fluid motion equations to enable much larger time steps. In these cases, closure models for the physical processes such as multiphase turbulence would have to account for all scales of fluctuation motion.

Multiphase Flow Simulation of the Bayer Process Precipitator: The vehicle for the development of a reactive multiphase flow simulation of the Bayer process precipitator was the LANL CFDLIB code library. The library solves mass, momentum and energy conservation equations for multiphase flows in complex geometries in two- and three-dimensions. CFDLIB has been used for a number of industrial projects concerning multiphase flow.

The development steps for this component of the project were:

1. Add functionality to CFDLIB for handling particle size distributions and weakly and directly coupled precipitation and agglomeration reaction kinetics. Add tracer variables for the computation of residence time distributions for the weakly coupled simulations. Implement precipitation and agglomeration kinetics into CFDLIB to enable directly coupled simulations. 
2. Identify and implement suitable multiphase flow physical models for exchange (drag, lift and added mass) and turbulence. Include effects of slip and exchange of turbulence kinetic energy among phases.

3. Validate simulations using physical models against suitable experimental data. Adjust physical models as needed. Pay particular attention to predicting the appropriate circulation patterns exhibited by many bubbly flows.

4. Perform non-reactive and reactive flow simulations for complex geometries representative of commercial alumina precipitators. Compare predictions of solids product size distribution, product yields and internal vessel profiles of velocities, temperatures and phase distribution to appropriate data sets.

Reaction Kinetics of the Bayer Process Precipitator: In this project component we addressed the modeling of the kinetics of the nucleation, growth and agglomeration of alumina particles that occurs in the alumina precipitator vessel. We additionally consider the coupling of these reactions to the multiphase flow simulation.

Our approach consisted of the following steps:

1. Evaluate and improve literature models for the kinetics of nucleation and growth of aluminum trihydroxide particles. The kinetics of this process has been described by the equation

$$
-\frac{d C_{t}}{d t}=k \exp \left(-\frac{14000}{R T}\right) A\left(C_{t}-C_{\infty}\right)^{2}
$$

where $C$ is the concentration of alumina in grams per liter, $k$ is a constant, $R$ is the universal gas constant, $T$ is the absolute temperature in $\mathrm{K}$ and $A$ is the seed surface area in meters squared per liter. The subscripts $t$ and $\infty$ respectively denote an arbitrary time $t$ and equilibrium [1]. The evaluation should strive to provide an explanation for the second power dependence on the concentration, for example.

2. Develop and validate a suitable model for the processes of agglomeration and breakage of aluminum trihydroxide particles. Start with the standard literature models. Investigate more fundamental approaches using collision theory and population balance master equations [5]. Improvements may be incorporated directly into CFDLIB for the direct coupling simulation. 
3. Develop a comprehensive predictive computational tool for simulating precipitation reaction kinetics including nucleation, growth and agglomeration for the weak coupling simulation.

Accomplishments: The following describes our accomplishments toward the goals outlined above. The results are of an intermediate nature due to the loss of funding after the first year.

Reactive Multiphase Flow Simulation: Progress toward the goals of this project outlined above for the reactive multiphase flow simulation were as follows:

Development of an algebraic slip model which enables the tracking of multiple particle sizes. In order to predict a useful particle size distribution for the alumina precipitator one typically must track and predict the motion of roughly 20 particle size classes in addition to the motion of the liquid and bubble phases. One way to do this is to treat each size class as a separate phase. This, however, can be computationally burdensome. As an alternative, we have eliminated the need to treat each size class as a separate phase by assuming no relative acceleration among the particle sizes. This reduces the individual particle size momentum balances to a set of algebraic equations which can be solved to yield the velocity of each individual size class relative to the average particle mixture velocity. A single momentum equation is then introduced for the particle mixture and the resulting computational burden is reduced. This technique has proved successful in both one-dimensional test calculations (results agreed well with the analytical solutions) and in a two-dimensional simulation of a three-phase precipitator flow.

Computation of residence time distributions from non-reactive simulations. In order to perform the weakly-coupled reactive multiphase flow simulation of the alumina precipitator, residence time distributions of the liquid and solid phases in the precipitator vessel were needed. The computational apparatus for this was installed into CFDLIB and residence times distributions were computed. This was done by simply transporting a passive scalar quantity with each phase in the calculation. After a quasi-steady state was obtained in the simulation, the value of the scalars were jumped from zero to one in the feed inlet. The value of the scalar variables in the outlet were recorded and served as a discrete residence time distribution. Such residence times could then be used to determine quantities such as short-circuiting and deadzone volumes for a non-ideal reactor model of the precipitator. Preliminary twodimensional simulations showed these techniques to be functioning properly and showing sensible results such as a time delay between the liquid and solid phase tracer signals in the outlet due to particle phase settling in the precipitator. 
Incorporation of Hounslow's [6] numerical algorithms for precipitation growth and agglomeration into CFDLIB for the coarsely resolved directly coupled simulations. In order to perform a coarsely resolved directly coupled reactive multiphase flow simulation, precipitation and agglomeration kinetics were incorporated into the CFDLIB multiphase flow code. This was done to explore an alternative to the weak-coupling calculation described above. To do this, the particle size distribution was treated in a discrete fashion with an arbitrary number of size classes.

Hounslow et. al. [6] have shown the proper way in which to treat such discrete particle size distributions. First, a geometrical discretization is typically desired so that the 'tails' of the particle size distributions can be adequately resolved. The diameters in such a geometric discretization typically increase from one size class to the next in a geometric fashion. For example, the diameter of adjacent size bins are often chosen to be in the ratio $2^{1 / 3}$ so that a doubling of volume occurs.

The geometric discretization introduces some anomalies which require attention to ensure accurate numerical treatment. In particular, one must preserve certain characteristics of the moment evolution of the particle size distribution called for by the continuous governing equations. Hounslow et. al. [6] were the first to provide the proper means of carrying this out in a practical numerical computation. The resulting numerical methods and equations of Hounslow et. al. [6] were incorporated directly in CFDLIB. Test cases presented in Hounslow et. al. [6] were successfully reproduced using the CFDLIB implementation. In addition, an alternative form of Hounslow's equations for the time rate of change of size class number density was discovered that has a more appealing mathematical form with a clear physical interpretation.

Testing of a multiphase turbulence model for use in bubbly flows. Phase segregation is an extremely important aspect of multiphase turbulent flows, including alumina precipitators. One of the most common examples is that of 'gulf-streaming' in a vertical two-phase flow bubble column. In a bubble column, under a wide range of conditions, gas tends to collect in the center of the column and form a high velocity channel. The resultant bouyancy forces in the flow induce a strong circulation flow pattern with liquid-rich material moving down the periphery of the column and gas-rich material moving up the center. Such circulation patterns affect mixing, hold-up and contacting times between phases and therefore directly affect equipment performance. Such flow patterns are also found in draft-tube bubble columns such as the alumina precipitators and are therefore of immediate practical interest. 
Turbulent stresses in multiphase flow play a crucial role in the process of phase segregation and the resulting bouyancy-driven circulation. The local level of fluctuation motion or turbulence in each phase acts like a pressure whose gradients can produce relative phase motion. This is the mechanism by which gas tends to collect in the center of the bubble column in the above 'gulf-streaming' example. The intensity of turbulence can also affect the rates at which materials spread away from regions of local concentration. Finally, correlations among the components of the turbulent fluctuation motion can induce transport of momentum and thereby moderate the intensity of mean velocity gradients in the flow.

In order to predict these affects, we (B. A. Kashiwa and W. B. VanderHeyden) have constructed a new set of multiphase flow turbulence transport equations for use in CFDLIB. The model equations have proven successful in the simulation of 'gulf streaming' in bubble columns. After further testing and development, we hope to publish our results in an appropriate forum.

Improved Reaction Kinetics for Alumina Precipitators: We (B. K. Kendrick and A. Redondo) have developed a kinetics model for alumina precipitation which solves the population and mass balance equations including the mechanisms of growth, agglomeration and secondary nucleation. A discrete particle size distribution is used and the resulting set of coupled firstorder differential equations is solved using a fourth-order Runge-Kutta method. The parameters of the model were determined by a nonlinear least squares fit of the supersaturation and particle count to the experimental batch crystallizer data of Misra and White [7]. In order to obtain better agreement with the experimental data, a more general growth equation was developed which is equivalent to the standard second-order growth equation for high supersaturations but gives first-order kinetics for low supersaturations ( $<20$ grams/liter). The agglomeration model is that of Hounslow, et. al. [6] and utilizes a size-independent kernel. This model assumes that all particle sizes agglomerate at the same rate and that this rate is proportional to the growth rate. In order to explain the induction effects which are observed in the experimental batch crystallizer data, a new secondary nucleation model was developed. The secondary nucleation model includes two mechanisms: 1) nucleation of small nuclei on the surface of the seed particles, and 2) the removal of the small nuclei from the surface of the seed particles via binary collisions between the seed particles. By fitting the experimental batch crystallizer data, we have found that the nucleation rate (mechanism 1) appears to exhibit fourth order kinetics and that the collision rate (mechanism 2) is proportional to the square of the supersaturation. Our current secondary nucleation model accurately reproduces the 
induction curves of Misra and White [7] at temperatures of 60 and 75 Celcius. We have also looked at several different constant flow rates through our ideal continuous stirred tank reactor model and have found oscillations in the supersaturation and particle number. These oscillations are due to the induction effects in the secondary nucleation mechanism. The amplitude and frequency of these oscillations depend upon the operating conditions such as the flow rate, supersaturation, and temperature. We are continuing to refine the model and are currently writing up a detailed description of our current model for publication.

\section{Conclusions}

While some of the building blocks for the reactive multiphase flow simulation of an alumina precipitator were put in place, the ultimate 3-year goal was not reached due to cessation of funding after the first year of the project. Nevertheless, the project did generate interest from ALCOA and future collaborations may therefore arise in which the original goals of this project might be achieved. 


\section{References}

[1] "Critical Reports on Applied Chemistry, Volume 20, Production of Aluminum and Alumina," edited by A. R. Burkin, John Wiley \& Sons, New York, (1987).

[2] Misra, C. and E.T. White, "Kinetics of Crystallization of Aluminum Trihydroxide from Seeded Caustic Aluminate Solutions," Chemical Engineering Progress Symposium Series, Number 110, pp. 53-74, (1971).

[3] Hsieh, P. , "Air-Agitated Alumina Crystallizer," in "Reactive Multiphase Flow Simulation Workshop Summary," ed. by W. B. VanderHeyden, LA-UR-95-2194, (1995).

[4] Burton, J.J., "Nucleation Theory," in "Statistical Mechanics, Part A: Equilibrium Techniques," edited by B.J. Berne, Plenum, New York, (1977).

[5] Van Kampen, N.G., "Stochastic Processes in Physics and Chemistry," North Holland, Amsterdam, (1981)

[6] M.J. Hounslow, R.L. Ryall, and V.R. Marshall, AlChE J. 34, 1821 (1988)

[7] C. Misra and E.T White, Chem. Eng. Progr. Sympos. Ser. 110, 53 (1970). 